\title{
Grado de relación entre violencia familiar y conexiones interpersonales en progenitores de familia, Chachapoyas, Amazonas
}

\section{Degree of relationship between family violence and interpersonal connections in parents, Chachapoyas, Amazonas}

\author{
Jak Man Pizango Antich ${ }^{1}$
}

\begin{abstract}
RESUMEN
La investigación tuvo por objetivo determinar el grado de relación entre violencia familiar y conexiones interpersonales en los progenitores de familia de la Institución Educativa "Pedro Castro Alva" de Chachapoyas, 2017; la población estuvo circunscrita a 155 progenitores, cuya muestra obtenida mediante muestreo probabilístico fue de 64 progenitores de familia. El estudio de tipo descriptivo-correlacional, se aplicó una encuesta válida y confiable estadísticamente, para la variable violencia familiar $\alpha=0.97$ y para conexiones interpersonales $\alpha=0.87$. Los resultados obtenidos demuestran relación significativa entre violencia familiar y conexiones interpersonales $(\mathrm{p}<0.05, \mathrm{X} 2=33.83, \mathrm{r}=0.185)$, es decir existe una relación positiva entre las variables de estudio; así mismo, se encontró que el 3\% de los progenitores, presentó violencia psicológica grave y moderada y en el 44\% se inicia relación de abuso; en el 70\% de los progenitores no existe violencia física y en el $23 \%$ se inicia relación de abuso; el 3\% de los progenitores presentó violencia económica grave y en el 56\% se inicia relación de abuso; el 2\% de los progenitores presentó violencia sexual grave, moderada y leve; y el $81.3 \%$ presentan un nivel de cautela de conexiones interpersonales, esto quiere decir, que el individuo se encuentra en un nivel bajo y que el nivel de estrés es muy alto. En conclusión, el estudio determinó que existe correlación significativa positiva pero muy baja estadísticamente entre violencia familiar y conexiones interpersonales en los progenitores de familia.
\end{abstract}

Palabras clave: Violencia familiar, conexiones interpersonales

\begin{abstract}
The research aimed to determine the degree of relationship between family violence and interpersonal connections in the parents of the Educational Institutión "Pedro Castro Alva" of Chachapoyas, 2017; the population was limited to 155 parents, whose sample obtained by probability sampling was 64 parents. The descriptivecorrelational type study, used as a technique the survey and as an instrument a statistically valid and reliable questionnaire, for the variable family violence $\alpha=0.97$ and for interpersonal connections $\alpha=0.87$. The results obtained show a significant relationship between family violence and interpersonal connections $(\mathrm{p}<0.05, \mathrm{X} 2=$ $33.83, r=0.185)$, i.e. there is a positive relationship between the study variables; likewise, it was found that $3 \%$ of the parents presented severe and moderate psychological violence and $44 \%$ of them started abusive relationships; in $70 \%$ of the parents there was no physical violence and $23 \%$ of them started abusive relationships; $3 \%$ of the parents presented severe economic violence and 56\% started abusive relationships; $2 \%$ of the parents presented severe, moderate and mild sexual violence; and $81.3 \%$ present a level of caution of interpersonal connections, which means that the individual is at a low level and that the level of stress is very high. In conclusion, the study determined that there is a significant positive but statistically very low correlation between family violence and interpersonal connections in parents.
\end{abstract}

Keywords: Family violence, interpersonal connections

Bachiller en Educación. Facultad de Educación y Ciencias de la Comunicación de la Universidad Nacional Toribio Rodríguez de Mendoza de Amazonas. Correo electrónico:pizango2007@gmail.com 


\section{INTRODUCCIÓN}

Los estudios realizados por la Organización Mundial de la Salud (OMS, 2009) sobre la violencia familiar ponen de manifiesto que, a pesar del progreso alcanzado, la sociedad sigue fallando a las mujeres en los momentos clave de la vida, puesto que, la mayor proporción de gobiernos y formuladores de políticas lo consideran como un problema social de índole menor; sin embargo, desde los noventa los esfuerzos liderados por mujeres, especialistas y gobiernos se han duplicado, comprometiéndose con la sensibilización pública para mitigar dicho problema.

Al respecto, las investigaciones internacionales llevadas a cabo por la OMS (2009) determinan que la violencia contra la mujer es un problema común, grave y general de lo sospechado hasta la fecha; estudios revisados desde 1999 en 35 países indican que entre el 10 y $52 \%$ sufrieron maltrato físico por su pareja en algún espacio de su vida, entre el 10 y $30 \%$ sufrió violencia sexual y entre el 10 y $27 \%$ de los abusos sexuales declararon haber sido víctimas cuando eran niñas.

A nivel mundial, del 23 al 38\% de las mujeres han sufrido violencia física o sexual por parte de su pareja íntima y el $42 \%$ de ellas han sufrido lesiones (Montero, et al, 2016). Por ej., el estudio poblacional más reciente realizado en Finlandia, mostró que el $17 \%$ de las mujeres han sido abusadas por su pareja actual y el $42 \%$ por una pareja anterior, siendo las cifras respectivas para los hombres $16 \%$ y $22 \%$. Anualmente, aproximadamente uno de cada diez ciudadanos en el mundo es objeto de violencia, y las mujeres sufren con mayor frecuencia violencia familiar y violencia sexual y los hombres otras formas de violencia interpersonal. Además, solo el $10 \%$ de las víctimas, informan haber sido agredidas más de 10 veces durante sus vidas.

Aunque la violencia familiar es tan común como otros delitos violentos, sus costos para la sociedad y la prevención tienden a ser discutidos menos. Además de las lesiones agudas, se ha demostrado que la violencia familiar tiene varios efectos duraderos, como una peor salud funcional y autoinformada, dolor de espalda, dolor de estómago, dolor de cabeza, síntomas psicosomáticos (Siltala et al., 2020). Los problemas de salud mental más comunes relacionados con la violencia familiar son la depresión, la ansiedad y el trastorno de estrés postraumático. También se sabe que las víctimas de violencia familiar son más suicidas y tienen más problemas de abuso de sustancias que la población en general (Siltala et al., 2020)

En esa perspectiva, en el Perú, el panorama es idéntico al contexto internacional, en tal sentido, los estudios realizados por Orna (2013) sobre los tipos de violencia familiar según distritos de Lima metropolitana, detallan que en todos los distritos se dio este tipo de violencia siendo el más habitual la física $(65.6 \%)$ seguida de la psicológica $(24.9 \%)$ y otros tipos de violencia ocurren en un $9.6 \%$ (421/4407). De igual manera, el Instituto Nacional de Estadística e Informática (INEI) - Encuesta Demográfica y de Salud Familiar (2013) señala que la violencia con la familia se da tanto en la zona rural como en la urbana; así por ej., en la sierra y selva el promedio es de $74.1 \%$.

De igual manera, Orna (2013) revela que la violencia familiar en el Perú, van cada año en aumento sus cifras al igual que el número de denuncias por violencia familiar registradas; por ej., las ciudades de Tumbes, Piura, Lambayeque, La Libertad, las fiscalías provinciales mixtas y de familia registraron un alto número de denuncias, tan solo Lambayeque registró 34845 y Piura 31076 denuncias.

En tal realidad, también se encuentra inmersa la jurisdicción de la región Amazonas. El INEI en el 2017, registró que el $71.8 \%$ de las mujeres fue víctima de este daño por su pareja en el que se destaca que el $65.8 \%$ sufrió daño psicológico o verbal, el $37.9 \%$ físico y el $6.5 \%$ sexual.

\section{MATERIAL Y MÉTODO}

El diseño empleado fue el descriptivo correlacional.

La población estuvo constituido por 155 progenitores de familia de la Institución Educativa "Pedro Castro Alva" del distrito de Chachapoyas, región Amazonas, Perú. La muestra se conformó por 64 progenitores. El muestreo fue probabilístico.

Se aplicó una encuesta para evaluar la violencia familiar; la misma que constaba de 20 preguntas, que a median la violencia psicológica, física, económica y sexual; con una valoración de escala Likert por pregunta de: Siempre: 5; muy frecuente: 4; alguna ocasión: 3 , rara vez: 2 ; y nunca: 0 .

Se aplicó un test con 10 preguntas para evaluar las conexiones interpersonales, cuya valoración fue: Muy bien: 3; moderadamente: 2; un poco: 1; y nada bien: 0 .

El indicador estadístico que se utilizó para determinar la confiabilidad del instrumento fue el estadístico Alfa de Cronbach, en el que se obtuvo un índice de confiabilidad del 0.97 para el test de violencia familiar y el 0.87 para el test que evaluó las conexiones interpersonales. 


\section{RESULTADOS}

Tabla 1

Distribución de niveles de violencia familiar.

\begin{tabular}{lccc}
\hline \multicolumn{1}{c}{ Niveles } & Frecuencia & Porcentaje & $\begin{array}{c}\text { Porcentaje } \\
\text { válido }\end{array}$ \\
\hline No existe violencia & 7 & 10,9 & 10,9 \\
Inicia relación de abuso & 50 & 78,1 & 78,1 \\
Violencia leve & 5 & 7,8 & 7,8 \\
Violencia moderada & 2 & 3,1 & 3,1 \\
\hline Total & 64 & 100,0 & 100,0 \\
\hline
\end{tabular}

En la tabla 1, se presenta los niveles de violencia familiar en los progenitores de familia, se encontró que en el $10.9 \%$ de los encuestados no existe violencia y el $78.1 \%$ inicia relación de abuso; encontrándose en violencia leve y moderada el 7.8 y $3.1 \%$ respectivamente.

\section{Tabla 2}

Distribución de los niveles de violencia familiar y dimensiones

\begin{tabular}{cccccccccc}
\hline \multirow{2}{*}{ Nivel de violencia } & \multicolumn{2}{c}{ Psicológica } & \multicolumn{2}{c}{ Frica } & \multicolumn{2}{c}{ Econ ómi ca } & \multicolumn{2}{c}{ Sexual } \\
& & $\mathrm{Fi}$ & $\%$ & $\mathrm{fi}$ & $\%$ & $\mathrm{fi}$ & $\%$ & $\mathrm{fi}$ & $\%$ \\
\hline No existe violencia & 0 & 24 & $38 \%$ & 45 & $70 \%$ & 14 & $22 \%$ & 59 & $92 \%$ \\
Inicia relación de abuso & $1-5$ & 28 & $44 \%$ & 15 & $23 \%$ & 36 & $56 \%$ & 2 & $3 \%$ \\
Violencia leve & $6-10$ & 8 & $13 \%$ & 0 & $0 \%$ & 9 & $14 \%$ & 1 & $2 \%$ \\
Violencia moderada & $11-15$ & 2 & $3 \%$ & 1 & $2 \%$ & 3 & $5 \%$ & 1 & $2 \%$ \\
Violencia grave & $16-20$ & 2 & $3 \%$ & 3 & $5 \%$ & 2 & $3 \%$ & 1 & $2 \%$ \\
\hline Total & 64 & $100 \%$ & 64 & $100 \%$ & 64 & $100 \%$ & 64 & $100 \%$ \\
\hline
\end{tabular}

Fuente: encuesta realizada, I.E. Pedro Castro, Chachapoyas.

La tabla 2, presenta frecuencias porcentuales en las dimensiones de la variable violencia familiar. En ella se observa que la mayor proporción de violencia grave se da en el tipo de violencia física, seguido de la violencia económica. Así también, se observa que en el $92 \%$ y el $70 \%$ no existe violencia física y sexual respectivamente, seguido del $38 \%$ y $22 \%$ en psicológica y económica. También se observa que en la mayor proporción de los entrevistados se inicia relación de abuso, siendo el $44 \%$ de psicológica, $23 \%$ física, $56 \%$ económica y el $3 \%$ de violencia sexual.

\section{Tabla 3}

Distribución de frecuencias de conexiones interpersonales que experimentan los progenitores defamilia.

\begin{tabular}{lccc}
\hline Cautela & 52 & 81,3 & 81,3 \\
Vulnerable & 8 & 12,5 & 12,5 \\
Diestro & 3 & 4,7 & 4,7 \\
Óptimo & 1 & 1,6 & 1,6 \\
\hline Total & 64 & 100,0 & 100,0 \\
\hline
\end{tabular}

En la tabla 3, se presenta las conexiones interpersonales que los progenitores de familia experimentan en la Institución Educativa Pedro Castro Alva; se observa que 52 padres de familia $(81.3 \%)$ presentan un nivel de cautela, esto quiere decir, que el individuo se encuentra en un nivel bajo y que el nivel de estrés es muy alto. Así mismo, el 12\% presentó un nivel vulnerable, lo que quiere decir que el individuo se encuentra en un estado de fragilidad, el ambiente que lo rodea lo afecta; por otro lado, el $4.7 \%$ presentó un nivel diestro y el solo el 1.6 un nivel óptimo. En el nivel diestro, el individuo es hábil en el manejo de sus emociones y las relaciones con los demás y en el nivel óptimo, el individuo se encuentra en un nivel alto de destreza emocional.

\section{Tabla 4}

Estadísticos descriptivos según escala Likert de las variables y dimensiones.

\begin{tabular}{lrrrrrrrrr}
\hline Variables y dimensiones & $\mathbf{N}$ & Rango & Mín. & Máx. & Media & $\begin{array}{c}\text { Desviación } \\
\text { estándar }\end{array}$ & Varianza \\
\hline Violencia psicológica & 64 & 19,00 &, 00 & 19,00 & 3,063 &, 54092 & 4,32738 & 18,726 \\
Violencia fisica & 64 & 20,00 &, 00 & 20,00 & 1,672 &, 56390 & 4,51120 & 20,351 \\
Violencia económica & 64 & 20,00 &, 00 & 20,00 & 4,484 &, 53730 & 4,29837 & 18,476 \\
Violencia sexual & 64 & 20,00 &, 00 & 20,00 &, 766 &, 41532 & 3,32256 & 11,039 \\
Violencia familiar (VF) & 64 & 62,00 &, 00 & 62,00 & 9,984 & 1,5123 & 12,09813 & 146,365 \\
Conexiones & 64 & 30,00 &, 00 & 30,00 & 12,172 &, 86245 & 6,89963 & 47,605 \\
interpersonales(Cl) & & & & & & & & & \\
N válido (por lista) & 64 & & & & & & &
\end{tabular}

En la tabla 4, se muestran los descriptivos de las variables y dimensiones, y se puede observar que los puntajes obtenidos para la variable VF fueron: Media=9.984, Desviación Estándar=12.098 y la Varianza de 146.365. De igual modo para la variable CI, los puntajes obtenidos fueron: Media $=12.172$, Des. Estándar=6,899 y la Varianza de 47.605. De igual manera fueron los puntajes obtenidos para cada una de las dimensiones de ambas variables.

\section{Tabla 5}

Prueba ji-Cuadrado según las variables violencia familiar y conexiones interpersonales, asi como dimensiones en progenitores de familia.

\begin{tabular}{|c|c|c|c|c|c|c|c|}
\hline Variables & Estadistico & calculado & tabuhr & $\mathrm{gl}$ & p-valor * & Decisión & Conclusión \\
\hline $\begin{array}{l}\text { Violencia familiar y } \\
\text { conexiones } \\
\text { interpersonales }\end{array}$ & $\mathrm{x}^{2}$ & 33,834 & 21.03 & 12 & 0.001 & Rechazar Ho & $\begin{array}{c}\text { Existe } \\
\text { relación } \\
\text { significativa } \\
\end{array}$ \\
\hline Dimensiones & Estadistico & calculado & tabuhar & $\mathrm{gl}$ & p-valor * & Decisión & Conclusión \\
\hline $\begin{array}{l}\text { Violencia familiar } \\
\text { psicológica }\end{array}$ & & 34,347 & 21.03 & 12 & 0.000 & Rechazar Ho & $\begin{array}{c}\text { Existe } \\
\text { relación } \\
\text { significativa }\end{array}$ \\
\hline $\begin{array}{l}\text { Violencia familiar } \\
\text { fisica }\end{array}$ & $x^{2}$ & 66,790 & 21.03 & 12 & 0.000 & Rechazar Ho & $\begin{array}{c}\text { Existe } \\
\text { relación } \\
\text { significativa }\end{array}$ \\
\hline $\begin{array}{l}\text { Violencia familiar } \\
\text { económica }\end{array}$ & & 37,919 & 21.03 & 12 & 0.000 & Rechazar Ho & $\begin{array}{c}\text { Existe } \\
\text { relación } \\
\text { significativa }\end{array}$ \\
\hline $\begin{array}{l}\text { Violencia familiar } \\
\text { sexual }\end{array}$ & & 67,061 & 21.03 & 12 & 0.000 & Rechazar Ho & $\begin{array}{c}\text { Existe } \\
\text { relación } \\
\text { significativa }\end{array}$ \\
\hline
\end{tabular}


La tabla 5, presenta la relación de las variables violencia familiar y conexiones interpersonales, encontrando que existe relación significativa estadísticamente, mediante la prueba ji-cuadrado (pvalor $<0.05$ ); así mismo para las dimensiones de la variable violencia familiar expresadas en violencia psicológica, física, económica y sexual, también se encontró que existe relación significativa estadísticamente mediante la prueba ji-cuadrada $(\mathrm{p}<0,05)$.

De igual manera, se evaluó las Correlaciones $\mathrm{R}$ de Rho de Spearman de las variables violencia familiar, conexiones interpersonales y dimensiones, en el que se encontró que hay una relación positiva muy baja de $\mathrm{r}=0,185$, así mismo la relación de las dimensiones de la VF: psicológica, física, económica y sexual, con las conexiones interpersonales, existe un nivel de correlación positiva muy baja $\mathrm{r}=0.128, \mathrm{r}=-0.111$, $\mathrm{r}=0.095 \mathrm{y} \mathrm{r}=0.173$ respectivamente; lo cual podemos concluir que existe una relación positiva muy baja estadísticamente entre dimensiones y variables haciendo el cruce de todas las dimensiones y variables del estudio.

\section{Figura 1}

Relación entre violencia familiar vs conexiones interpersonales en progenitores de familia.

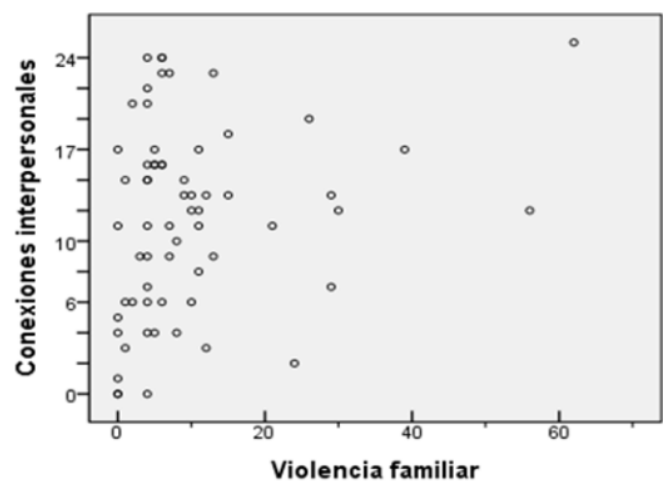

En la figura 1, se puede observar gráficamente que existe relación significativa entre la violencia familiar y conexiones interpersonales, lo cual, dicha relación es positiva muy baja estadísticamente.

\section{DISCUSIÓN}

Se encontró que existe relación entre la violencia familiar y conexiones interpersonales, sin embargo, dicha relación es positiva pero muy baja estadísticamente $r=0.185$, estos hallazgos pueden compararse con Plasencia et al. (2017), quienes al correlacionar las fortalezas y las áreas del funcionamiento familiar encontraron que el optimismo con el factor involucramiento afectivo disfuncional dio un $\mathrm{r}=0.54 \mathrm{y}$ auto-regulación con factor involucramiento afectivo disfuncional el $\mathrm{r}=0.54$, cifras ligeramente mayor a la nuestra, cuyas variables son semejantes a tal investigación.

Con respecto a las dimensiones de la variable VF, estas se correlacionaron con las conexiones interpersonales, y se encontró que existe un nivel de correlación positiva muy baja $\mathrm{r}=0.128, \mathrm{r}=-0.111$, $\mathrm{r}=0.095$ y $\mathrm{r}=0.173$ respectivamente; Plasencia et al. (2017), al correlacionar las dimensiones de violencia familiar con las fortalezas humanas, encontraron que el factor de involucramiento afectivo disfuncional mostró el mayor número de correlaciones con las fortalezas de mentalidad abierta, coraje, creatividad, perseverancia, autorregulación y optimismo; resultados diferentes y mayores a la nuestra. De igual manera, Smiley et al. (2020) encontró que los niños se vieron más afectados que las niñas, con un $62 \%$ después que sufrieron abuso físico en la infancia en comparación con el $59 \%$ de las niñas. Los niños también enfrentan niveles más altos de abuso emocional que las niñas. 1 de cada 5 niñas y 3 de cada 10 niños sufren abuso emocional por parte de un padre o pariente. Las niñas, por otro lado, enfrentan tasas mucho más altas de explotación y abuso sexual que los niños. Aproximadamente el 14\% y el $22 \%$ de las niñas informan haber sufrido explotación sexual y abuso sexual, respectivamente. En comparación con los niños, el 7\% y el 6\% de los cuales informan haber experimentado explotación sexual y abuso sexual.

En esa misma lógica, en la presente investigación se analizó en términos porcentuales las frecuencias con las que se dieron los tipos de violencia según las dimensiones de la variable VF y se encontró que en mayor proporción de los entrevistados se inicia relación de abuso, siendo el $44 \%$ de psicológica, $23 \%$ física, $56 \%$ económica y el $3 \%$ de violencia sexual. Tales resultados se comparan con López y Lozano (2017) quienes muestran que el 35\% de las mujeres de todo el mundo ha sido víctima de violencia física y/o sexual en algún momento de su vida. En Perú, el $28 \%$ de mujeres y el 40 de niños sufrieron violencias de parte de las personas con quienes conviven. Por su parte, Psaki et al. (2017) utilizando datos longitudinales de un estudio sobre escolaridad y adolescencia, investigaron las asociaciones entre la violencia escolar y doméstica y tres resultados educativos: ausentismo, aprendizaje y deserción. La mitad de los encuestados había experimentado violencia doméstica y violencia de género entre los 18 y los 21 años. Las asociaciones entre violencia y educación fueron mixtas: la violencia sexual relacionada con la escuela se asoció con peores resultados educativos posteriores para los hombres $\mathrm{y}$, en menor medida, para las mujeres; la violencia doméstica se asoció con un mayor ausentismo de los hombres y la posterior deserción de las mujeres; y la violencia física se asoció con un menor ausentismo y un mejor desempeño subsecuente en aritmética para las mujeres. 
Según el INEI (2017) reporta que al año 2016, el $68,2 \%$ de mujeres de 15 a 49 años de edad han sufrido violencia. Tales resultados se relaciones los nuestros, pues que se encontró que la mayor proporción de tipo violencia grave fue en la violencia física (5\%), seguido de la violencia económica (3\%). Un informe sobre la prevención del maltrato infantil realizado en 40 países europeos, que examinó las tasas de experiencia de varios tipos de violencia familiar entre niños menores de 18 años, indicó que el 22,9\% de los participantes había experimentado violencia física y el 29,1\% había experimentado violencia psicológica. Otra encuesta reciente realizada en los Estados Unidos encontró que la tasa de violencia física por parte de un cuidador durante el último año fue del 5\% para la muestra completa, y la tasa de violencia física por parte de un cuidador durante la vida fue del $18,1 \%$ para el grupo de $14-17$ años. Las tasas de violencia psicológica por parte de un cuidador durante el último año fueron del 9,3\% para la muestra completa y del $23,9 \%$ durante la vida para el grupo de 14 a 17 años (Haj-Yahia et al., 2019).

Por otro lado, Nava-Navarro, Onofre-Rodríguez, y Báez-Hernández, (2017) encontraron que el 43\% de la muestra en estudio presentó autoestima baja, de ella, el $63.2 \%$ han sufrido violencia de pareja. Tales resultados son cifras altas a las nuestras y difieren el tipo de análisis. Por su parte, Jaen-Cortés, et al. (2015) encontraron que los factores asociados a la violencia familiar, fueron: escolaridad, violencia durante la infancia, la interrupción de un embarazo, entre otros. Contrario a tales resultados, Sala et al. (2010) encontraron que el 23,5\% de las mujeres maltratadas por su pareja presentaba enfermedad psiquiátrica, el $15 \%$ de las no maltratadas presentaron este tipo de enfermedad y el $44 \%$ de las maltratadas (de estas, en el $68 \%$ la enfermedad era posterior al maltrato). Los resultados de la presente investigación coinciden con González-Alexander et al. (2013). Tales autores reportan que la violencia física es un hecho concreto, su negación dificulta obtener indicadores precisos de su frecuencia y prevalencia, los porcentajes obtenidos muestran una diferencia significativa en los reactivos utilizados. En consecuencia, tales resultados coinciden cualitativamente con la nuestra.

Los resultados de diferentes investigaciones, tales como los desarrollado por Haj-Yahia et al., (2019) afirman que los niños que han estado expuestos a la violencia familiar pueden no desarrollar relaciones estrechas con sus compañeros y es más probable que experimenten dificultades en el funcionamiento social. La exposición a la violencia familiar (presenciar, experimentar o ambos) está relacionada con el desarrollo de problemas de comportamiento externalizantes (como comportamiento agresivo y delictivo, problemas de conducta) y problemas de comportamiento internalizantes (como comportamiento retraído y síntomas de ansiedad y depresión, que podría aumentar el riesgo de ser rechazado por sus compañeros. La exposición a la violencia familiar también tiene un efecto destructivo en la relación entre padres e hijos y, como resultado, limita considerablemente los recursos de apoyo disponibles para el niño dentro de la familia. Los niños que crecen en un hogar con un padre que es una fuente potencial de peligro y que a menudo es dañino, no disponible e impredecible, no logran desarrollar una confianza básica en los demás y es probable que formen vínculos inseguros con sus cuidadores

Finalmente, la revisión de antecedentes ha permitido encontrar que los niños que experimentan algún tipo de violencia familiar demuestran más problemas de comportamiento y emocionales que los niños que no han tenido tales experiencias. Algunos de estos niños exteriorizan sus reacciones negativas $y$, en consecuencia, desarrollan problemas notables (p. Ej., Mal comportamiento, comportamiento agresivo, abuso de sustancias, abandono de la escuela, etc.), mientras que algunos de ellos internalizan sus sentimientos y, como resultado, pueden desarrollar una alta niveles de depresión y ansiedad. Los estudios también han encontrado que experimentar violencia parental está asociado con quejas somáticas, salud física más baja, problemas sociales, problemas cognitivos y bajo rendimiento académico. Se descubrió que los adultos que habían experimentado diferentes formas de violencia, abuso y/o negligencia de los padres durante la infancia tenían un mayor riesgo de desarrollar trastornos alimentarios, adicción al alcohol, abuso de drogas y mala salud en general, así como síntomas de depresión y ansiedad (Haj-Yahia et al., 2019).

\section{CONCLUSIONES}

Se encontró mediante la prueba ji-cuadrado (p-valor $<0.05$ ) que existe relación significativa entre violencia familiar y conexiones interpersonales.

Se encontró que el 3\% de los progenitores de familia presentó violencia psicológica grave y moderada, el $13 \%$ violencia leve, y en el $44 \%$ se inicia relación de abuso, y sólo en el $38 \%$ no existió este tipo de violencia.

Se encontró que en el 70\% de los progenitores de familia, no existe violencia familiar física y en el $23 \%$ se inicia relación de abuso. Sin embargo, se encontró un $5 \%$ de violencia grave.

Se encontró que el 3\% de los progenitores de familia, presentó violencia familiar económica grave, el 5\% moderada, el 14\% violencia leve, y en el 56\% inicia relación de abuso; sin embargo, en el $22 \%$ no existe violencia.

Se encontró que el $2 \%$ de los progenitores de familia, presentó violencia familiar sexual grave, moderada y 
leve; en el $3 \%$ se inicia relación de abuso y en el $92 \%$ no existe este tipo de violencia.

Las conexiones interpersonales que los progenitores de familia experimentan; se caracterizan porque el $81.3 \%$ presentan un nivel de cautela, el $12 \%$ presentan un nivel vulnerable, el $4.7 \%$ presentan un nivel diestro y el solo el $1.6 \%$ presentan un nivel óptimo.

Respecto al grado de asociación entre las dimensiones de violencia familiar, expresadas en violencia psicológica, física, económica, sexual y conexiones interpersonales que experimentan los progenitores de familia, hay una relación significativa positiva $p<0,05$, (violencia familiar psicológica $\mathrm{r}=0.095$, física $\mathrm{r}=0.128$, económica $\mathrm{r}=$ 0.111 y sexual $r=0.173$ ) con las conexiones interpersonales, pero muy baja estadísticamente.

\section{REFERENCIAS BIBLIOGRÁFICAS}

González, E., Ponce, E. R., Landgrave, S., Baillet, L. E., Munguía, S., y Jáuregui, O. (2013). Frecuencia, factores de riesgo y tipos de violencia intrafamiliar en un grupo de mujeres de una clínica de medicina familiar en la ciudad de México. Atención Familiar, 20(3), 86-90. https://doi.org/10.1016/S1405-8871 (16)3009

Haj-Yahia, M. M., Sokar, S., Hassan-Abbas, N., \& Malka, M. (2019). The relationship between exposure to family violence in childhood and post-traumatic stress symptoms in young adulthood: The mediating role of social support. Child Abuse and Neglect, 92, 126-138. https://doi.org/10.1016/j.chiabu.20 19.03.023

Instituto Nacional de Estadística e Informática Encuesta Demográfica y de Salud Familiar (2013). Violencia contra las mujeres, niñas y niños. INEI. https://goo.gl/nDBHkv

Instituto Nacional de Estadística e Informática-INEI (2017). Perú: indicadores de violencia familiar y sexual, 2000-2017. https://bit.ly/3i4Z6jD

Jaen-Cortés, C, Aragón, S. R., Amorin, E. F., \& Rivera, L. (2015). Violencia de Pareja en Mujeres: Prevalencia y Factores Asociados. Acta de Investigación Psicológica, 5(3), 2224-2239. https://doi.org/10.1016/S2007-47 19(16)30012-6

López, G. y Lozano, M. (2017). La violencia familiar: situación actual y recomendaciones para su prevención en la ciudad de Iquitos. Fondazione L'Albero della Vita. Home

Montero, E., Delis, M. T., Ramírez, R., Milán, A. L. y Cárdenas, R. (2016). Realidades de la violencia familiar en el mundo contemporáneo. MEDISAN, 15 (4), 515-525. https://bit.ly/35nw $\mathrm{gWe}$

Nava, V., Onofre, D., \& Báez, F. (2017). Autoestima, violencia de pareja y conducta sexual en mujeres indígenas. Enfermería Universitaria, 14(3), 162-169. https://bit.ly/2MEd5Rv

Organización Mundial de la Salud-OMS (2009). Las mujeres y la salud. Ginebra, Suiza: Organiza ción Mundial de la Salud. https://bit.ly/3hXg VAP

Orna, O. (2013). Factores determinantes de la violencia familiar y sus implicancias: análisis de los estudios estadísticos sobre la Violencia Familiar en el distrito de San Juan de Lurigancho (Lima), Callao y otras ciudades del país. (Tesis de maestría; Universidad Nacional Mayor De San Marcos). https://goo.gl/Ms3m

Plasencia, M. L., Eguiluz, L. de L., \& Osorio, M. (2017). Relación entre la dinámica familiar y las fortalezas humanas. Journal of Behavior, Health \& Social Issues, 8(2), 1-8. https://doi. org/10.1016/j.jbhsi.2016.11.001

Psaki, S. R., Mensch, B. S., \& Soler-Hampejsek, E. (2017). Associations between violence in school and at home and education outcomes in rural Malawi: A longitudinal analysis. Comparative Education Review, 61(2), 354-390. https://doi. org/10.1086/691117

Sala, I., Hernández, A. R., Ros, R., Lorenz, G., \& Parellada, N. (2010). Violencia doméstica: preguntar para detectar. Atención Primaria / Sociedad Española de Medicina de Familia y Comunitaria, 42(2), 70-7. https://doi.org/10.10 16/j.aprim.2009.04.007

Siltala, H. P., Kuusinen-Laukkala, A., \& Holma, J. M. (2020). Victims of family violence identified in emergency care: Comparisons of mental health and somatic diagnoses with other victims of interpersonal violence by a retrospective chart review. Preventive Medicine Reports, 19, 101136. https://doi.org/10.1016/j.pmedr.2020 .101136 
Smiley, A., Moussa, W., Ndamobissi, R., \& Menkiti, A. (2020). The negative impact of violence on children's education and well-being: Evidence from Northern Nigeria. International Journal of Educational Development, 81, 102327. https://doi.org/10.1016/j.ijedudev.2020.10232 